\title{
Galectin-1 overexpression promotes progression and chemoresistance to cisplatin in epithelial ovarian cancer
}

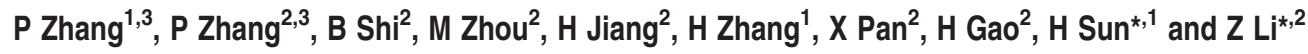

This study was performed to investigate the role of galectin-1 (Gal-1) in epithelial ovarian cancer (EOC) progression and chemoresistance. Tissue samples from patients with EOC were used to examine the correlation between Gal-1 expression and clinical stage of EOC. The role of Gal-1 in EOC progression and chemoresistance was evaluated in vitro by siRNA-mediated knockdown of Gal-1 or lentivirus-mediated overexpression of Gal-1 in EOC cell lines. To elucidate the molecular mechanisms underlying Gal-1-mediated tumor progression and chemoresistance, the expression and activities of some signaling molecules associated with Gal-1 were analyzed. We found overexpression of Gal-1 in advanced stages of EOC. Knockdown of endogenous Gal-1 in EOC cells resulted in the reduction in cell growth, migration, and invasion in vitro, which may be caused by Gal-1's interaction with H-Ras and activation of the Raf/extracellular signal-regulated kinase (ERK) pathway. Additionally, matrix metalloproteinase-9 (MMP-9) and c-Jun were downregulated in Gal-1-knockdown cells. Notably, Gal-1 overexpression could significantly decrease the sensitivities of EOC cells to cisplatin, which might be ascribed to Gal-1-induced activation of the H-Ras/Raf/ERK pathway and upregulation of p21 and Bcl-2. Taken together, the results suggest that Gal-1 contributes to both tumorigenesis and cisplatin resistance in EOC. Thus, Gal-1 is a potential therapeutic target for EOC.

Cell Death and Disease (2014) 5, e991; doi:10.1038/cddis.2013.526; published online 9 January 2014

Subject Category: Cancer

Epithelial ovarian cancer (EOC) is the most common cause of death from gynecologic tumors worldwide, and overall survival rates have improved very little in the past decade. ${ }^{1}$ One of the reasons for the poor survival rate is that most EOC cases are diagnosed at an advanced stage. High-grade serous EOC is frequently associated with intraperitoneal spread and distant metastases. The standard treatment is debulking surgery followed by paclitaxel- and platinum-based chemotherapy. After platinum-based chemotherapy, 25\% of patients will develop resistance to the therapeutics within 6 months, ${ }^{2}$ and the overall 5 -year survival rate is $\sim 40 \%{ }^{3}$ Unfortunately, the mechanisms underlying cisplatin resistance in EOC are not yet fully understood.

Galectins are galactoside-binding glycoproteins with a conserved carbohydrate recognition domain. ${ }^{4}$ Galectin-1 (Gal-1) is considered to be the prototypical galectins and is expressed in many tumor types such as astrocytoma, melanoma, and prostate, thyroid, colon, bladder, and ovarian cancers. ${ }^{5,6}$ Increasing clinical evidence has confirmed that Gal-1 is involved in cancer progression and is associated with a poor prognosis in head and neck, prostate, lung, and ovarian cancers. ${ }^{7-11} \mathrm{Gal}-1$ participates in tumor progression by evoking immunosuppression through the induction of activated T-cell apoptosis, transformation, angiogenesis, and metastasis. ${ }^{12,13}$

In this study, we investigated the role of Gal-1 in EOC progression and cisplatin resistance and studied the downstream effects of Gal-1 deregulation. We found that Gal-1 was highly expressed in EOC tissues and its tumor levels were positively correlated with clinical stage. Gal-1 can promote the growth, migration, and invasion of EOC cells and their resistance to cisplatin. Furthermore, we revealed that Gal-1 could upregulate c-Jun, matrix metalloproteinase-9 (MMP-9), $\mathrm{Bcl}-2$, and p21 expression, possibly through activation of the H-Ras/Raf/extracellular signal-regulated kinase (ERK) pathway.

\section{Results}

Gal-1 overexpression in EOC is associated with poor prognosis. Gal-1 expression has been correlated with poor EOC prognosis. ${ }^{11}$ To further confirm this relationship, we analyzed Gal-1 expression in normal human ovary and EOC tissue by quantitative real-time PCR (qRT-PCR) (Figure 1a),

\footnotetext{
${ }^{1}$ Department of Gynecology, Obstetrics and Gynecology Hospital of Fudan University, Shanghai Key Laboratory of Female Reproductive Endocrine Related Diseases, Shanghai, China and ${ }^{2}$ State Key Laboratory of Oncogenes and Related Genes, Shanghai Cancer Institute, Renji Hospital, Shanghai Jiaotong University School of Medicine, Shanghai, China

${ }^{*}$ Corresponding author: H Sun, Obstetrics and Gynecology Hospital of Fudan University, 419, Fang Xie Road, Shanghai 200011 , China. Tel: +86 2133189900 ; Fax: +86 21 63455050; E-mail: hongsun57@ hotmail.com

or Z Li, State Key Laboratory of Oncogenes and Related Genes, Renji Hospital, Shanghai Jiaotong University School of Medicine, No. 25/Ln2200, XieTu Road, Shanghai 200032, China. Tel: +86 21 64436601; Fax: +86 21 64432027; E-mail: zonghaili@ shsmu.edu.cn

${ }^{3}$ These authors contributed equally to this work.

Keywords: galectin-1; epithelial ovarian cancer; progression; chemoresistance

Abbreviations: EOC, epithelial ovarian cancer; ERK, extracellular signal-regulated kinase; MMP-9, matrix metalloproteinase-9

Received 6.9.13; revised 22.11.13; accepted 25.11.13; Edited by A Stephanou
} 

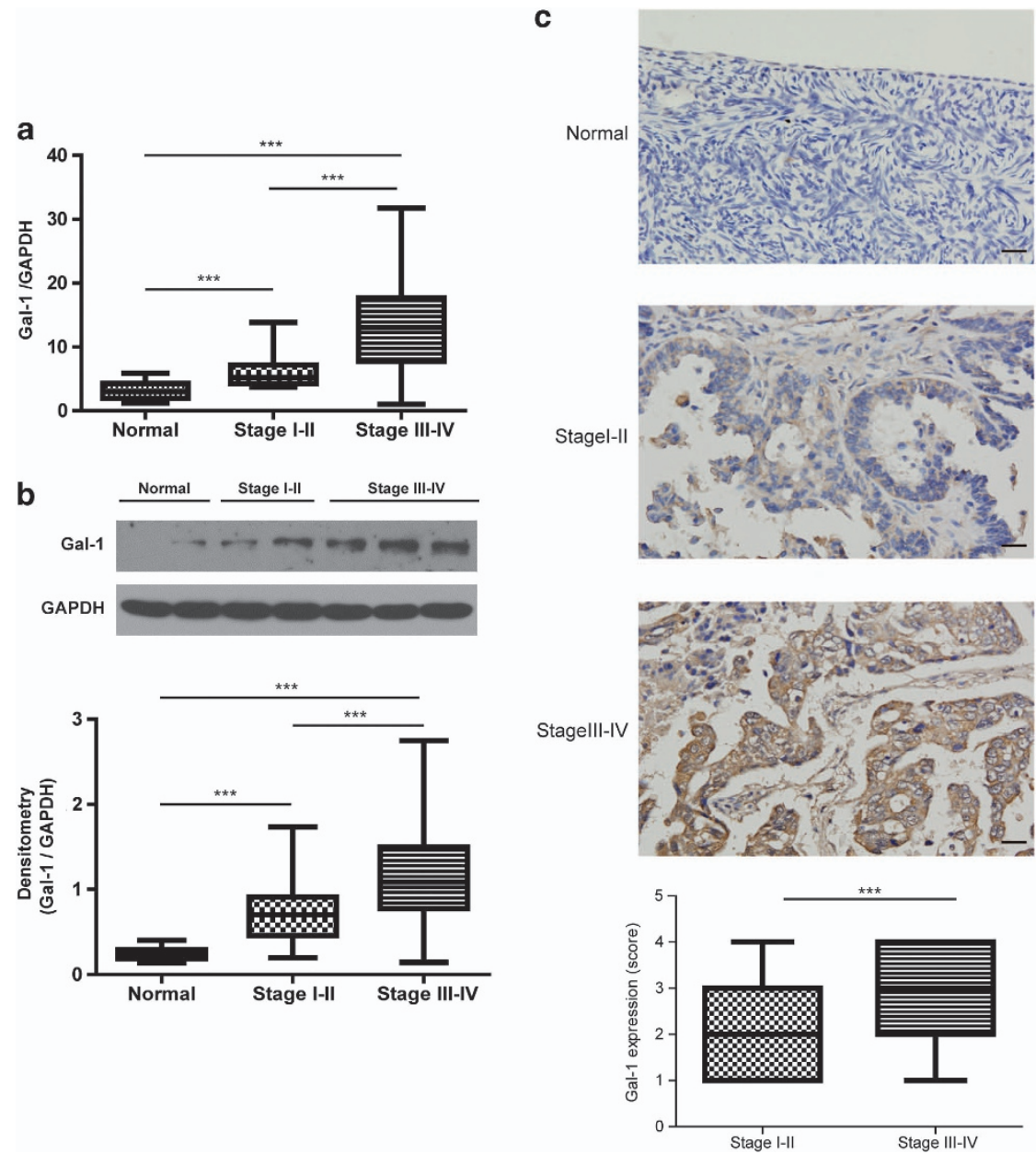

Figure 1 High Gal-1 expression is positively correlated with advanced stage and poor prognosis in EOC. (a) The mRNA levels of Gal-1 in fresh-frozen specimens from normal human ovary and EOC tissues were detected by qRT-PCR. (b) Gal-1 protein in fresh-frozen specimens from normal human ovary and EOC tissues was detected by western blotting (top); GAPDH was used as a loading control; densitometric quantitation of the western blots is also shown (bottom). (c) Immunohistochemical staining was conducted using an antibody against Gal-1 on paraffin-embedded specimens (top); magnification, $\times 200$; scale bars, $100 \mu$ m; Gal-1 expression levels were quantified according to the immunostaining score (bottom). ${ }^{\star \star \star} P<0.001$

western blotting (Figure 1b), and immunohistochemistry (Figure 1c). At the mRNA and protein level, Gal-1 expression was observed in EOC tissues. In contrast, in normal ovarian tissue, little or no Gal-1 expression was observed. Gal-1 expression in early-stage (I-II) and advanced-stage (III-IV) EOC tissues was significantly higher than in normal ovarian tissues $(P<0.0001)$. In addition, Gal-1 expression in advanced-stage (III-IV) was significantly higher than in early-stage $(I-I I)$ EOC tissues $(P<0.0001)$ (Figures 1a-C). Importantly, when EOC patients were divided into two groups according to staining intensity of immunohistochemistry, namely a low expression group (score $\leq 2)$ and a high expression group (score $\geq 3$ ), the progression-free survival (PFS) in the high Gal-1 expression group was shorter than that in the low Gal-1 expression group $(P=0.008)$ (Figure 2).

Gal-1 is involved in EOC cell proliferation, migration, and invasion in vitro. To elucidate the role of Gal-1 in EOC progression, Gal-1 siRNA was used to reduce Gal-1 expression in the human EOC SKOV3ip1 cell line, which has a high level of Gal-1 protein expression (Figure 3a). Both
Gal-1 siRNA1 and siRNA2 significantly reduced Gal-1 expression (Figure $3 \mathrm{~b}$ ), as well as the proliferation $(P<0.01)$ (Figure 3c), migration, and invasion of SKOV3ip1 cells $(P<0.01)$ (Figure $3 d$ ). siRNA1 was slightly more effective than siRNA2. To further evaluate whether Gal-1 upregulation could promote tumor proliferation, migration, and invasion, lentivirus-mediated delivery of Gal-1 cDNA was used to increase Gal-1 expression in the human EOC Hey cell line, which has low Gal-1 protein expression (Figure 3a). Upregulation of Gal-1 expression was observed in Gal-1 infectants (relative to the GFP control) (Figure 3e). Gal-1 upregulation significantly increased the proliferation $(P<0.01)$ (Figure 3f), migration, and invasion abilities of Hey cells compared with the GFP control $(P<0.01)$ (Figure 3g).

Gal-1 upregulates p-Raf-1 and p-ERK expression by interacting with H-Ras in EOC cells. Gal-1 is a critical scaffolding protein and a major regulator of $\mathrm{H}$-Ras nanoclusters, and it contributes to Ras membrane anchorage and tumor transformation. ${ }^{14}$ It has been reported that intracellular 


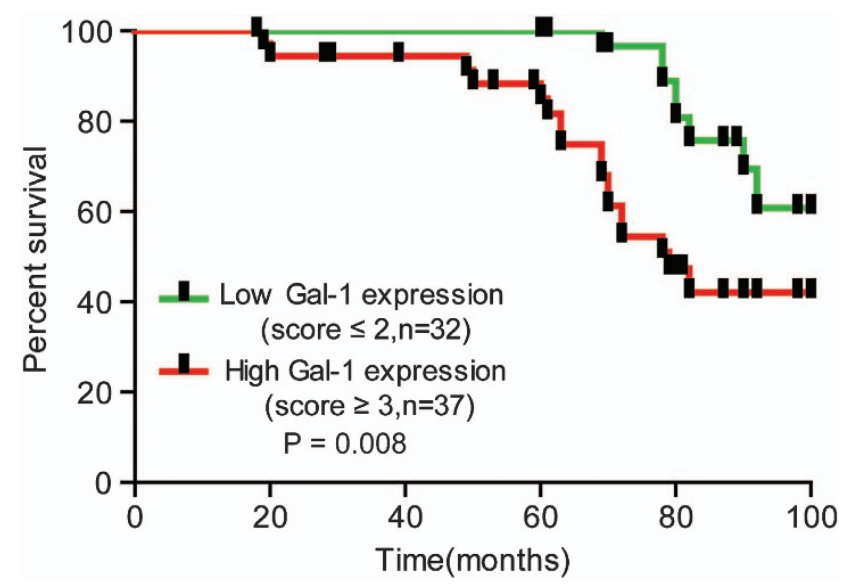

Figure 2 Progression-free survival curves of patients according to the Gal-1 immunostaining score in EOC

Gal-1 mediates different biological functions through its interaction with H-Ras. ${ }^{15,16}$ Our results showed that Gal-1 downregulation in SKOV3ip1 cells decreased H-Ras, p-Raf-1, and $p$-ERK expression, whereas Gal-1 upregulation in Hey cells increased $\mathrm{H}$-Ras, $\mathrm{p}$-Raf-1, and $\mathrm{p}$-ERK expression. No significant difference in p-Akt expression was observed with changes in Gal-1 expression (Figure 4a). To further confirm the interaction of $\mathrm{Gal}-1$ with $\mathrm{H}$-Ras, co-immunoprecipitation followed by western blotting was performed and indicated that Gal-1 directly interacted with H-Ras (Figure 4b).

Gal-1 promotes EOC cell proliferation, migration, and invasion by upregulating the expression of $c$-Jun and MMP-9. c-Jun, an important component of the AP-1 complex, is frequently overexpressed in many tumor types and has been confirmed to promote cellular proliferation, migration, and angiogenesis. ${ }^{17}$ MMP-9 is expressed in ovarian epithelial tumor cells and has a critical role in cancer invasion and migration. ${ }^{18,19}$ Our results indicated that siRNAmediated Gal-1 downregulation in SKOV3ip1 cells potently reduced the expression of MMP-9 and c-Jun, whereas Gal-1 overexpression in Hey cells increased the expression of MMP-9 and C-Jun (Figures 4c and d). MMP-2 expression in EOC cells (A2780/CP20) has been shown to be regulated by Gal-1. ${ }^{11}$ However, no marked difference in MMP-2 expression was observed in SKOV3ip1 following treatment with Gal-1 siRNA or Hey cells transfected with Gal-1 (Figures 4c and d).

Gal-1 overexpression in EOC cells enhances their resistance to cisplatin. Gal-1 knockdown has been reported to sensitize lung cancer cells to platinum-based chemotherapy (cisplatin). ${ }^{16}$ To investigate the effects of Gal-1 on the chemoresistance of EOC cells, A2780/CP, a well-established cisplatin-resistant cell line, was used to investigate whether Gal-1 participated in the chemoresistance of EOC cells. Both siRNA1 and siRNA2 against Gal-1 significantly downregulated Gal-1 expression in A2780/CP cells (Figure 5a). Control siRNA-, Gal-1 siRNA1-, and Gal-1 siRNA2-transfected A2780/CP cells were treated with or without cisplatin. Cisplatin inhibited cell growth (Figure 5b) and induced apoptosis (Figure 5c; Supplementary Table 3) in these cell lines in a dose-dependent manner. Notably, A2780/CP cells transfected with Gal-1 siRNA1 and Gal-1 siRNA2 were significantly more sensitive to cisplatin than those transfected with control siRNA $(P<0.01$ or $P<0.001)$. The effect of siRNA1 was slightly better compared with siRNA2, corresponding to their efficacies in Gal-1 suppression. To further confirm the role of Gal-1 in the cisplatin resistance of EOC cells, Hey cells transfected with Gal-1 or GFP were also treated with or without cisplatin. Interestingly, in the presence of cisplatin, Gal-1 transfectants exhibited a lower sensitivity to cisplatin than GFP transfectants $(P<0.01)$ (Figures $5 d$ and e; Supplementary Table 3$)$. Moreover, A2780/CP cells treated with Gal-1 siRNA demonstrated reduced expression of $\mathrm{H}$-Ras, p-Raf-1, p-ERK (Figure 6a), p21, and Bcl-2 (Figure 6b). In contrast, Gal-1 overexpression in Hey cells increased the expression of $\mathrm{H}$-Ras, p-Raf-1, p-ERK (Figure 4a), p21, and Bcl-2 (Figure 6b). Knockdown of Gal-1 expression in A2780/CP cells reduced p21 expression, whereas Gal-1 overexpression in Hey cells increased p21 expression, which was primarily observed in the cytoplasm, with little found in the nucleus (Figures 6c and d).

\section{Discussion}

In this study, we demonstrated that intracellular Gal-1 could promote EOC progression, chemoresistance to cisplatin, and increase H-Ras, p-Raf-1, p-ERK, MMP-9, c-Jun, p21, and Bcl-2 expression.

Compared with normal ovarian tissues, Gal-1 was overexpressed in EOC tissues and tumor levels of Gal-1 were positively correlated with clinical stage, suggesting that Gal-1 participates in EOC progression. Moreover, a high level of Gal-1 expression correlated with poor prognosis in EOC. Similar results have been reported previously. ${ }^{11,20}$

$\mathrm{H}$-Ras/Gal-1 interactions are essential for the membrane anchorage of $\mathrm{H}-\mathrm{Ras}$, and intracellular Gal-1 stabilizes the interactions of $\mathrm{H}$-Ras-GTP with the cell membrane, which then triggers a kinase cascade comprising Raf and ERK. ${ }^{14,21}$ In agreement with these previous findings, our study also demonstrated a direct interaction between $\mathrm{Gal}-1$ and $\mathrm{H}$-Ras in SKOV3ip1 and Hey cells. Furthermore, we observed the activation of Raf-1 and ERK but not the AKT pathway. C-Jun, a transcription factor, has been linked to cell proliferation, tumor cell survival, and apoptosis. Many human tumors exhibit elevated levels of c-Jun expression, and c-Jun activation can be a critical factor for transformation and tumorigenesis. ${ }^{22}$ c-Jun protein is activated by a phosphorylation cascade that requires activation of MEK-ERK and $\mathrm{JNK}^{23}$ In this study, we found that Gal-1 overexpression upregulated c-Jun expression in EOC cells. Thus, the Gal-1-induced upregulation of p-Raf-1, p-ERK, and c-Jun may be a major contributing factor to the proliferation of EOC cells. MMP-9 proteolytically acts on the extracellular matrix (ECM) and activates signals involved in tumor cell migration and invasion. ${ }^{24,25}$ Studies in ovarian carcinoma cells have shown that ERK1 and ERK2 are important for fibronectin-stimulated invasiveness and MMP-9 secretion. ${ }^{26}$ Additionally, activation of ERK leads to increased expression and secretion of MMP-9. ${ }^{27}$ In our study, 


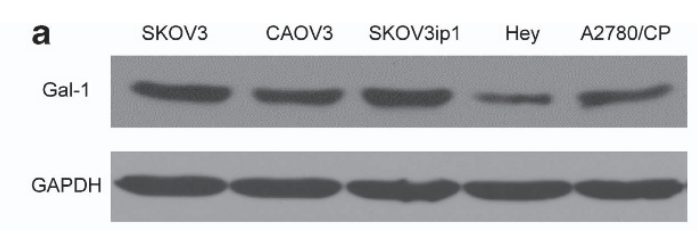

b

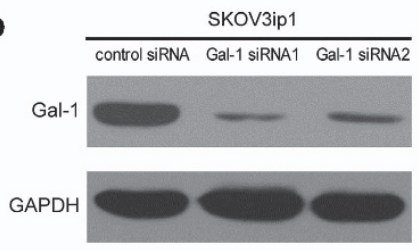

C

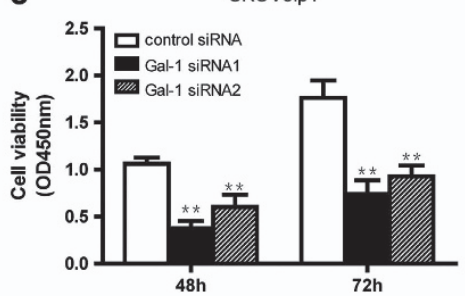

d

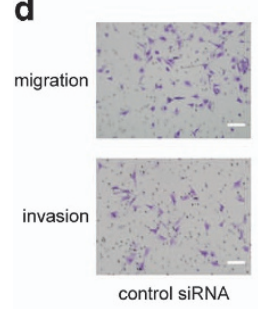

SKOV3ip1

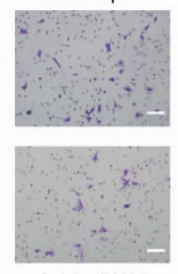

Gal-1 siRNA1

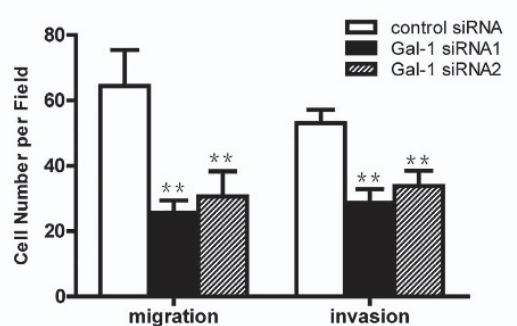

e

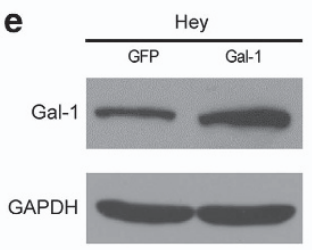

f

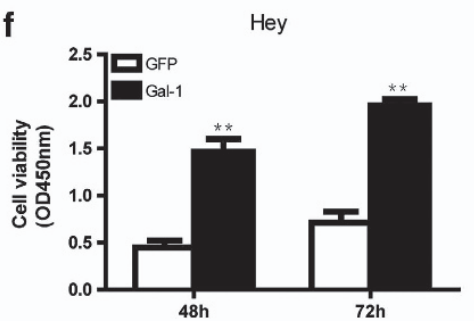

g

Hey
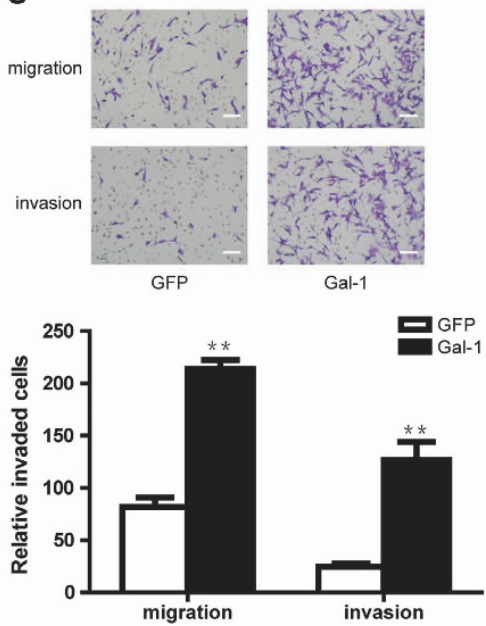

Figure 3 Gal-1 promotes the proliferation, migration, and invasion of EOC cells. (a) Gal-1 expression in several EOC lines was examined by western blotting. GAPDH was used as the loading control. (b) Knockdown of Gal-1 expression in SKOV3ip1 cells were verified by western blotting. GAPDH was used as a loading control. (c) Knockdown of Gal-1 expression in SKOV3ip1 cells reduced cell proliferation. (d) Knockdown of Gal-1 expression in SKOV3ip1 cells reduced cell migration and invasion. Magnification, $\times 200$. Scale bars, $100 \mu \mathrm{m}$. (e) Gal-1 overexpression in Hey cells was verified by western blotting. GAPDH was used as a loading control. (f) Overexpression of Gal-1 in Hey cells increased cell proliferation. (g) Overexpression of Gal-1 in Hey cells increased cell migration and invasion. Magnification, $\times 200$. Scale bars, $100 \mu \mathrm{m}$. ${ }^{* *} P<0.01$

Gal-1 knockdown in SKOC3ip1 cells reduced p-Raf-1, p-ERK, and MMP-9 expression, whereas Gal-1 overexpression in Hey cells upregulated $p$-Raf- $1, p-E R K$, and MMP-9 expression. Taken together, these data suggest that intracellular Gal-1 promotes EOC cell invasion and migration possibly through the Raf-1/ERK/MMP-9 pathway. Further experiments are needed to confirm the link between Gal-1 effects on EOC cell invasion and migration and Gal-1 modulation of the Raf-1/ ERK/MMP-9 pathway.

Cisplatin-based chemotherapies are used as the first-line treatment for ovarian cancers. Although there is often a high responsiveness at first, the majority of patients eventually progress with platinum-resistant disease. Cytoplasmic p21 expression is related to drug resistance in several cancers. ${ }^{28}$
Additionally, ERK activation has been strongly implicated in p21 induction. ${ }^{29}$ The proto-oncogene Bcl-2 has been discovered in several tumors as a critical regulator of apoptosis. ${ }^{30-33}$ Among the $\mathrm{Bcl}-2$ protein family members, it has been repeatedly shown that $\mathrm{Bcl}-2$ overexpression delays the onset of apoptosis induced by several cytotoxic drugs. Substantial research has shown that downregulation of Bcl-2 expression can recover the sensitivity of cancer cells to anticancer drugs. ${ }^{34}$ Activation of the ERK pathway can also protect pancreatic tumor cells from apoptosis by regulating the expression of Bcl-2. ${ }^{35}$ Here, we found that Gal-1 knockdown sensitized EOC cells to cisplatin, whereas Gal-1 overexpression reduced the sensitivity of EOC cells to cisplatin. Moreover, Gal-1 knockdown reduced the expression of H-Ras, 

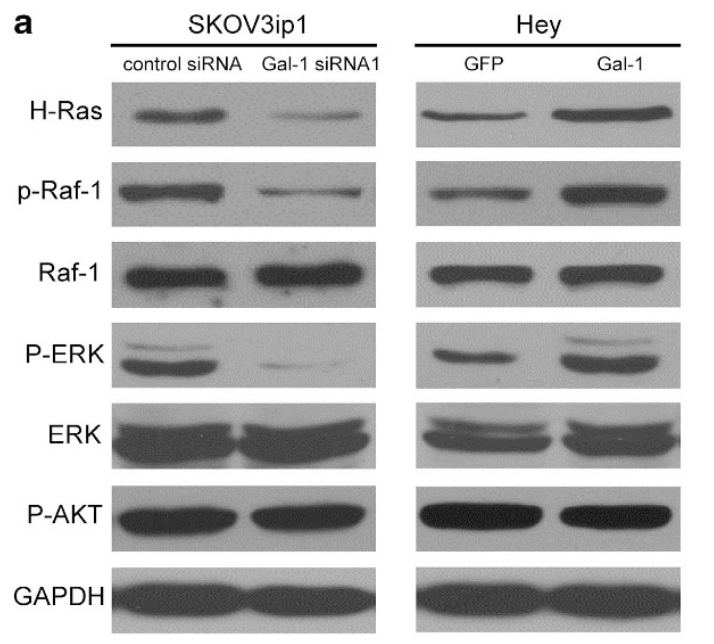

b
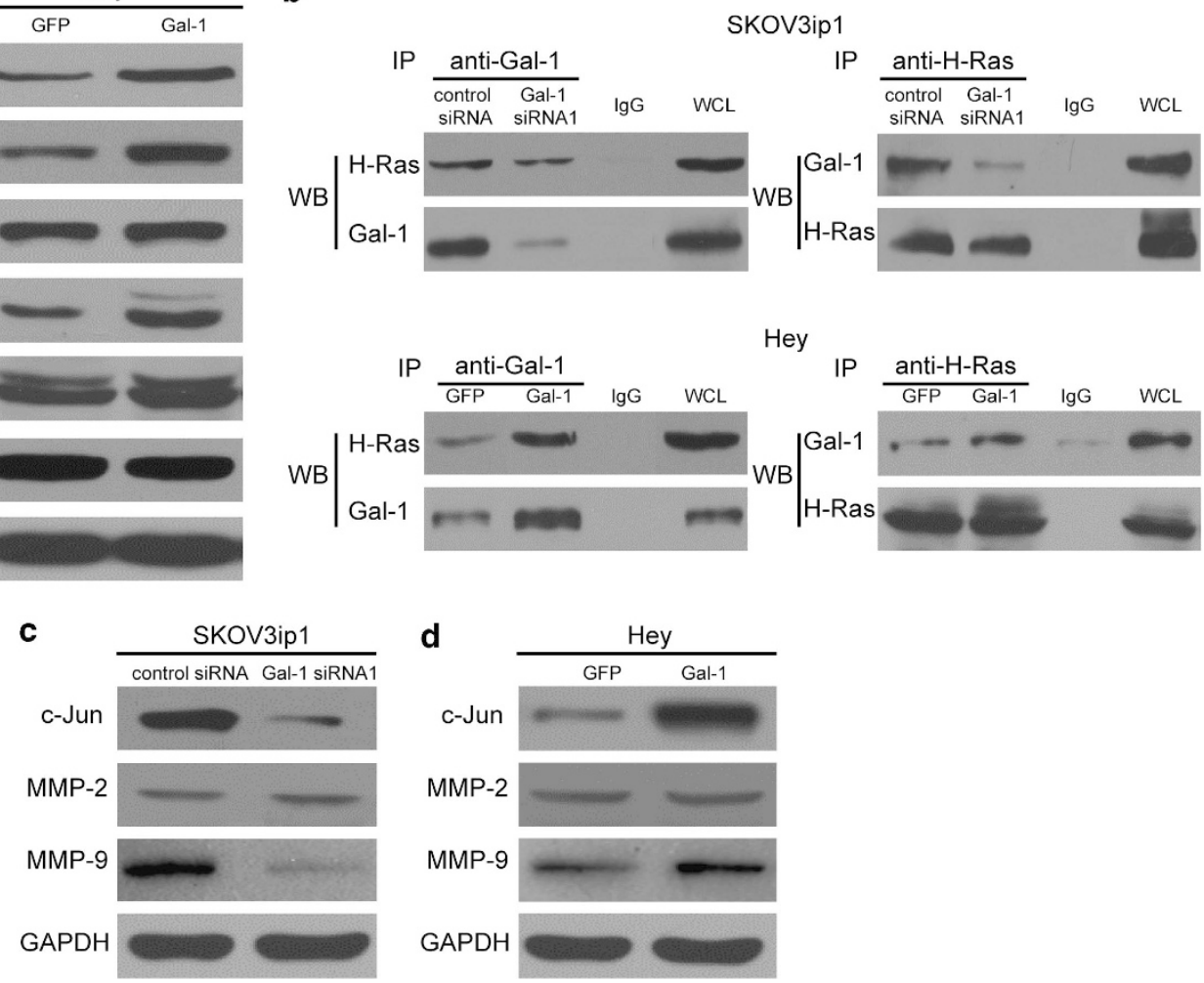

Figure 4 Gal-1 expression level affects the activities of the H-Ras/Raf-1/ERK pathway. (a) Protein levels of H-Ras/Raf-1/ERK in Gal-1 siRNA1- and control siRNAtransfected SKOV3ip1 cells and GFP/Gal-1-transfected Hey cells were detected by western blotting. GAPDH was used as a loading control. (b) The interaction of Gal-1 and $\mathrm{H}$-Ras was examined by immunoprecipitation (IP); IgG was used as the negative control. WCL, whole-cell lysate control. (c) Protein levels of MMP-9, C-Jun, and MMP-2 in Gal-1 control siRNA- and siRNA1-transfected SKOV3ip1 cells. GAPDH was used as a loading control for western blotting. (d) Protein levels of MMP-9, c-Jun, and MMP-2 in GFP/Gal-1-transfected Hey cells. GAPDH was used as a loading control for western blotting

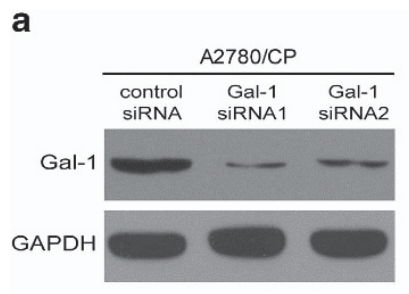

b
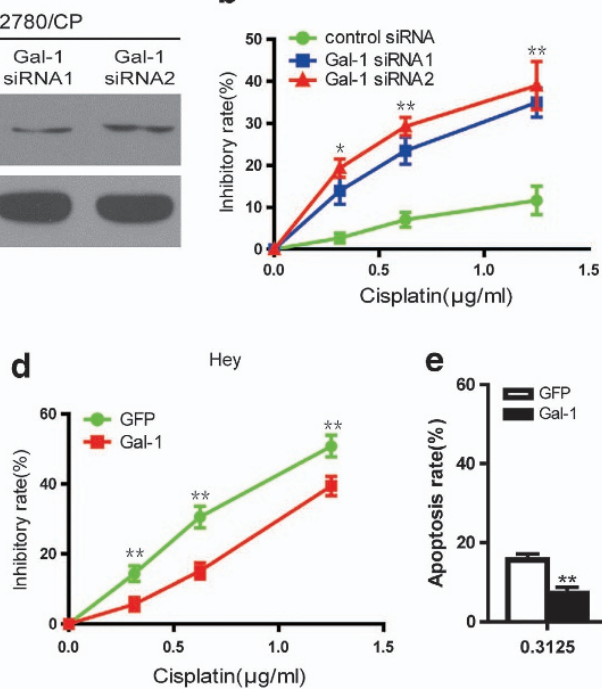

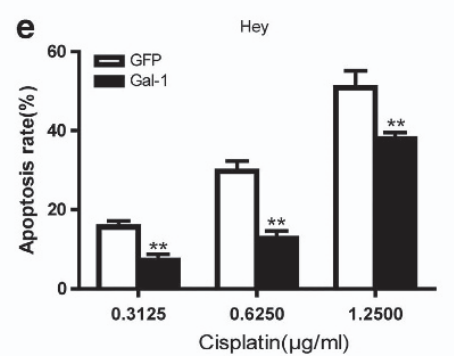

Figure 5 Gal-1 overexpression contributes to cisplatin resistance of EOC cells. (a) Knockdown of Gal-1 expression in A2780/CP cells by Gal-1 siRNA1 and siRNA2 was verified by western blotting. GAPDH was used as a loading control. (b) A2780/CP cells transfected with control siRNA, Gal-1 siRNA1, or Gal-1 siRNA2 were treated with different concentrations of cisplatin $(0.3125,0.625$, and $1.25 \mu \mathrm{g} / \mathrm{ml})$ for $72 \mathrm{~h}$. The cell inhibitory rate was quantified by the CCK-8 assay. (c) The cell apoptosis rates of siRNA1transfected and siRNA2-transfected A2780/CP cells in the presence of different concentrations of cisplatin $(0.3125,0.625$, and $1.25 \mu \mathrm{g} / \mathrm{ml})$ were measured by flow cytometry. (d) The growth inhibition rate of Hey cells transfected with GFP or Gal-1 in the presence of different concentrations of cisplatin was quantified by the CCK-8 assay. (e) The cell apoptosis rate of Gal-1-transfected Hey cells in the presence of different concentrations of cisplatin was detected by flow cytometry. ${ }^{\star} P<0.05,{ }^{* \star} P<0.01,{ }^{\star \star \star} P<0.001$ 
a

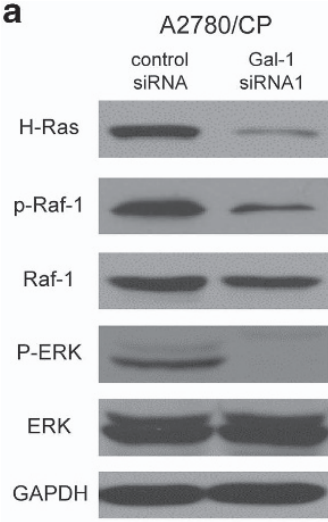

b

p21

Bcl-2

GAPDH

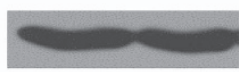

Hey

GFP Gal-1

p21

$\mathrm{Bcl}-2$

GAPDH
C
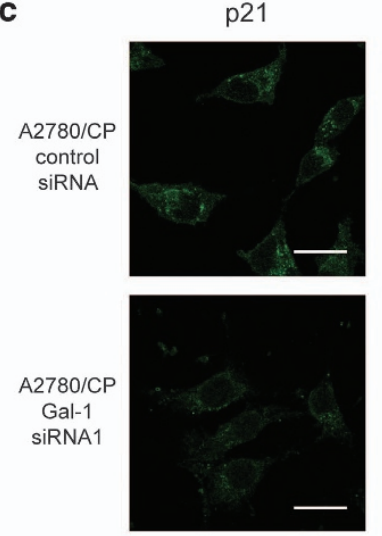

d
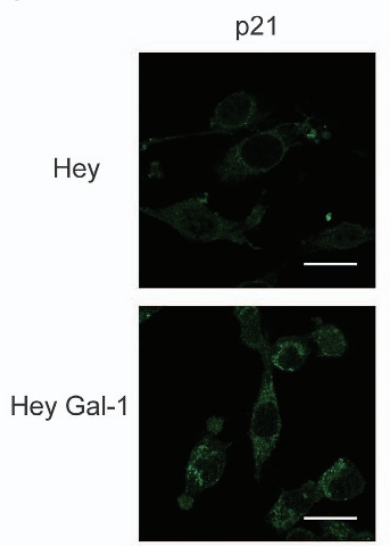
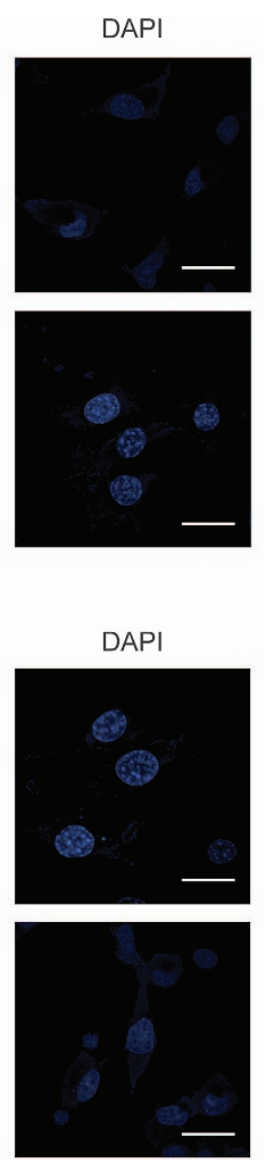
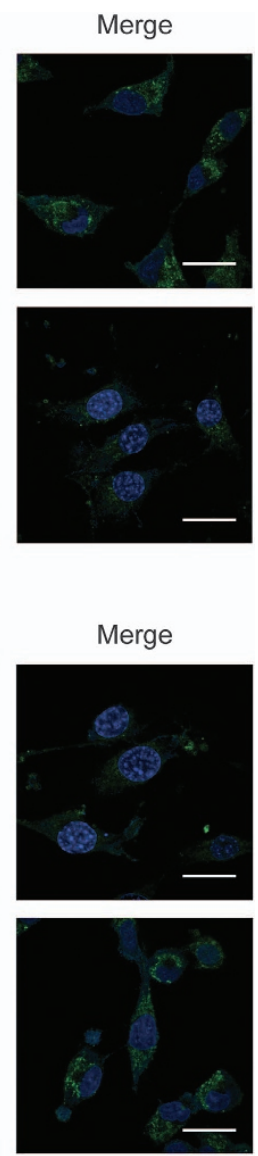

Figure 6 Gal-1 mediates activation of the H-Ras/Raf-1/ERK pathway and increased expression of p21 and Bcl-2. (a) Knockdown of Gal-1 expression in A2780/CP cells reduced H-Ras, p-Raf-1, and p-ERK expression. GAPDH was used as a loading control. (b) Knockdown of Gal-1 expression in A2780/CP cells reduced p21 and Bcl-2 expression, whereas overexpression of Gal-1 in Hey cells increased p21 and Bcl-2 expression. GAPDH was used as a loading control. (c) p21 expression was detected by immunofluorescence staining in A2780/CP cells treated with Gal-1 siRNA1 or control siRNA. Green fluorescence: p21; DAPI staining for nuclear DNA; Scale bar, $40 \mu$ m. (d) p21 expression was detected by immunofluorescence staining in Hey cells transfected with Gal-1 or GFP expression cassettes. Green fluorescence: p21; DAPI staining for nuclear DNA; Scale bar, $40 \mu \mathrm{m}$

p-Raf-1, p-ERK, p21, and Bcl-2. In contrast, Gal-1 overexpression activated the $\mathrm{H}$-Ras/Raf-1/ERK pathway and upregulated the expression of p21 and Bcl-2. Together, these data indicate that Gal-1 contributes to cisplatin resistance in EOC cells, which might be ascribed to Gal-1-mediated activation of the H-Ras/Raf-1/ERK pathway. Further experiments are needed to confirm the link between Gal-1 effects on cisplatin resistance, $\mathrm{H}$-Ras pathway and p21 and $\mathrm{Bcl}-2$.

In conclusion, our findings suggest that Gal-1 is an important player in the malignancy of EOC cells by promoting cell proliferation and invasion. Additionally, Gal-1 is involved in cisplatin resistance in EOC cells. Our data indicate that Gal-1 induces activation of the H-Ras/Raf-1/ERK pathway and has an important role in EOC progression and cisplatin resistance. Thus, Gal-1 may be considered as a potential therapeutic target for delaying EOC progression and increasing cisplatin sensitivities.

\section{Materials and Methods}

Cell culture. The human EOC cell lines SKOV3, SKOV3ip1, CAOV3, and Hey and the cisplatin-resistant EOC cell line A2780/CP were purchased from American Type Culture Collection (ATCC, Manassas, VA, USA). All cell lines were maintained in Dulbecco's modified Eagle's medium (DMEM) supplemented with $10 \%$ fetal bovine serum and antibiotics in a humidified atmosphere of $95 \%$ air and $5 \% \mathrm{CO}_{2}$ at $37^{\circ} \mathrm{C}$.

Clinical samples. Fresh-frozen specimens of human normal ovary tissues $(n=17)$ and primary EOC tissues $(n=82)$ obtained along with written informed consent and pathology reports from the Obstetrics and Gynecology Hospital of Fudan University (Supplementary Table 1) were used for qRT-PCR and western blotting. Paraffin-embedded specimens of normal human ovaries $(n=10)$ and EOC tissues $(n=69)$ obtained from the Obstetrics and Gynecology Hospital of Fudan University (Supplementary Table 2) were used for immunohistochemistry. Sample collection was performed after receiving approval from the institutional ethics review committee of the Obstetrics and Gynecology Hospital of Fudan University. No patient had undergone chemotherapy before surgery. Surgical evaluation was used to determine the clinical stage and presence of metastases, whereas histopathologic analysis was performed by gynecologic pathologists to assess cancer type and grade.

Immunohistochemistry. To assess Gal-1 expression in tumors, formalinfixed, paraffin-embedded tumor tissues were immunostained using Gal-1 antibody (Santa Cruz, CA, USA). After deparaffinization and rehydration, the tissue sections were incubated with $3 \% \mathrm{H}_{2} \mathrm{O}_{2}$ in methanol to quench endogenous peroxidase activity. The sections were blocked for $30 \mathrm{~min}$ with $1 \%$ bovine serum albumin and incubated overnight with the primary antibody at $4^{\circ} \mathrm{C}$. Sections were then washed with PBS and incubated with an HRP-conjugated secondary antibody for $45 \mathrm{~min}$. 
The products were visualized using a diaminobenzidine staining kit (Tiangen Biotech, Beijing, China) and counterstained in hematoxylin. All the sections were observed and photographed with an Axioskop 2 microscope (Carl Zeiss, Oberkochen, Germany). To evaluate Gal-1 expression, yellow cytoplasmic staining was considered as positive. Staining intensity was assigned a score as follows: 0 , no staining; 1 , weak intensity; 2 , moderate intensity; 3 , high intensity; and 4 , very high intensity.

Quantitative real-time PCR. Total RNA was extracted with the TRIzol Kit (Invitrogen, Carlsbad, CA, USA) according to the manufacturer's instructions. qRTPCR was performed using a SYBR Green PCR kit (TaKaRa, Otsu, Japan), according to the manufacturer's instructions. The primer sequences used were as follows: Gal-1 forward primer 5'-CTCCTGACGCTAAGAGCTTCG-3', reverse primer 5'-CCAGGCTGGAAGGGAAAGAC-3'; GAPDH forward primer $5^{\prime}$-CC TGGCACCCAGCACAAT- $3^{\prime}$, reverse primer $5^{\prime}$-GGGCCGGACTCGTCATCG-3'.

Gal-1 knockdown with siRNA. Gal-1 siRNA oligonucleotides were transfected using Lipofectamine 2000 (Invitrogen) according to the manufacturer's instructions. The sequence of Gal-1 siRNA1 was 5'-UUGCUGUUGCACACGAUGGUGUUGG-3'. The sequence of Gal-1 siRNA2 was 5'-GCUGCCAGAUGGAUACGAAUUTGGA-3'.

Lentivirus production and transduction of target cells. The Gal-1 sequences were amplified by PCR, confirmed by sequencing, and then inserted into a pWPT vector (a generous gift from Dr T Didier, University of Geneva, Geneva, Switzerland) by replacing GFP to generate pWPT-Gal-1. To produce virus particles, $20 \mu \mathrm{g}$ of pWPT-Gal-1 was transfected with $15 \mu \mathrm{g}$ of packaging plasmid psPAX2 and $5 \mu \mathrm{g}$ of $\mathrm{G}$ protein of the vesicular stomatitis virus (VSV-G) envelope plasmid pMD2.G (generous gifts from Dr T Didier) into 293T cells using a calcium phosphate transfection system. Hey cells were transduced with recombinant lentiviral particles to produce polyclonal cells with stable expression of Gal-1 and confirmed by western blotting.

In vitro cell proliferation assay. The effect of the test agents on cell viability was assessed with the CCK-8 assay as previously described. ${ }^{36}$ Three independent experiments were performed.

Apoptosis assays in EOC cell lines. Apoptosis was assessed using an Annexin V-coupled fluorescein isothiocyanate (FITC) apoptosis detection kit-1 (BD Pharmingen, San Diego, CA, USA). A2780/CP Gal-1 siRNA and A2780/CP control siRNA cells were incubated for $72 \mathrm{~h}$ alone with varying concentrations of cisplatin (each at $0,0.3125,0.625$, and $1.25 \mu \mathrm{g} / \mathrm{ml}$ ). Briefly, cells were removed from a six-well plate by incubation with trypsin-EDTA, washed twice in PBS, and resuspended in $1 \mathrm{ml}$ of Annexin V-binding buffer at $10^{6} \mathrm{cell} / \mathrm{s} / \mathrm{ml}$. Annexin V-coupled FITC and propidium iodide were added (each at $5 \mu \mathrm{l}$ per $10^{5}$ cells). Samples were mixed gently, incubated for $15 \mathrm{~min}$ at room temperature in the dark, and then subjected to flow cytometry to evaluate the number of apoptotic cells. All experiments were repeated at least three times.

Immunoprecipitation and western blotting. The supernatant from whole-cell lysates was harvested and immunoprecipitated using the indicated primary antibodies or non-specific $\lg G$ as a negative control, which was precleared by protein $\mathrm{G}$ magnetic beads at $4^{\circ} \mathrm{C}$ overnight. Proteins were separated on 10 or $12 \%$ SDS-PAGE gels and transferred onto nitrocellulose membranes (Bio-Rad, Hercules, CA, USA). The following antibodies were purchased from Santa Cruz Biotechnology (Santa Cruz, CA, USA): mouse anti-human Gal-1, rabbit anti-human H-Ras, rabbit anti-human ERK, mouse anti-human $\mathrm{p}$-ERK, rabbit anti-human c-Jun, mouse anti-human MMP-9, rabbit anti-human p21, rabbit antihuman Bcl-2, and rabbit anti-human Raf-1 antibodies. Rabbit anti-human p-AKT (Ser473), rabbit anti-human p-Raf-1 (Ser338), and rabbit anti-human MMP-2 antibodies were purchased from Cell Signaling (Danvers, MA, USA). The mouse anti-GAPDH antibody was purchased from KangChen Bio-tech (Shanghai, China). All experiments were repeated at least twice.

Transwell migration and invasion assay. Cell migration and invasion were gauged using a Transwell migration assay and a Matrigel invasion assay $\left(8 \mu \mathrm{m}\right.$ pore size; BD Falcon, San Jose, CA, USA) as previously described. ${ }^{37}$

Immunofluorescence staining. Immunofluorescence staining was performed on cultures after fixing the cells in methanol for $15 \mathrm{~min}$. Non-specific binding was blocked with a 5\% BSA-phosphate buffer solution for $1 \mathrm{~h}$. The cells were then incubated with rabbit polyclonal antibodies specific for p21 diluted at $1: 100$ in blocking buffer overnight at $4^{\circ} \mathrm{C}$. After washing with PBS, the cells were incubated with secondary antibodies diluted at 1:500 in blocking buffer for $2 \mathrm{~h}$ at room temperature. The cells were washed with PBS and incubated with DAPI for $10 \mathrm{~min}$ at room temperature. Images were obtained on a confocal microscope (Carl Zeiss, Oberkochen, Germany).

Statistical analysis. All data are presented as the mean \pm SD. The data were examined using analysis of variance (ANOVA) and the least significant differences method for multisample comparisons or Student's $t$-test for twosample comparisons. Pearson's chi-square test and Fisher's exact test were used to assess the statistical significance of the association between Gal-1 expression and clinicopathologic parameters. Kaplan-Meier curves were plotted to assess the effects of Gal-1 expression on PFS. Survival curves were compared using the log-rank test. $P<0.05$ was considered as statistically significant.

\section{Conflict of Interest}

The authors declare no conflict of interest.

Acknowledgements. This study was supported by the research fund of the State Key Laboratory of Oncogenes and Related Genes (91-10-06) and the National Basic Research Program (grant no. 2010CB529902).

1. Vaughan S, Coward JI, Bast RC Jr, Berchuck A, Berek JS, Brenton JD et al. Rethinking ovarian cancer: recommendations for improving outcomes. Nat Rev Cancer 2011; 11: 719-725.

2. Miller DS, Blessing JA, Krasner CN, Mannel RS, Hanjani $P$, Pearl ML et al Phase II evaluation of pemetrexed in the treatment of recurrent or persistent platinumresistant ovarian or primary peritoneal carcinoma: a study of the Gynecologic Oncology Group. J Clin Oncol 2009; 27: 2686-2691.

3. Siegel R, Naishadham D, Jemal A. Cancer statistics 2012CA Cancer J Clin 2012; 62 : 10-29.

4. Ito K, Stannard K, Gabutero E, Clark AM, Neo SY, Onturk S et al. Galectin-1 as a potent target for cancer therapy: role in the tumor microenvironment. Cancer Metastasis Rev 2012; 31: 763-778.

5. Satelli A, Rao US. Galectin-1 is silenced by promoter hypermethylation and its re-expression induces apoptosis in human colorectal cancer cells. Cancer Lett 2011; 301: $38-46$.

6. Ito K, Ralph SJ. Inhibiting galectin-1 reduces murine lung metastasis with increased CD4 $(+)$ and CD8 $(+)$ T cells and reduced cancer cell adherence. Clin Exp Metastasis 2012; 29: 561-572.

7. van Kooyk Y, Rabinovich GA. Protein-glycan interactions in the control of innate and adaptive immune responses. Nat Immunol 2008; 9: 593-601.

8. Le QT, Shi G, Cao H, Nelson DW, Wang Y, Chen EY et al. Galectin-1: a link between tumor hypoxia and tumor immune privilege. J Clin Oncol 2005; 23: 8932-8941.

9. van den Brule FA, Waltregny D, Castronovo V. Increased expression of galectin-1 in carcinoma-associated stroma predicts poor outcome in prostate carcinoma patients. J Pathol 2001; 193: 80-87.

10. Szoke T, Kayser K, Baumhakel JD, Trojan I, Furak J, Tiszlavicz L et al. Prognostic significance of endogenous adhesion/growth-regulatory lectins in lung cancer. Oncology 2005; 69: 167-174.

11. Kim HJ, Jeon HK, Cho YJ, Park YA, Choi JJ, Do IG et al. High galectin-1 expression correlates with poor prognosis and is involved in epithelial ovarian cancer proliferation and invasion. Eur J Cancer 2012; 48: 1914-1921.

12. Camby I, Le Mercier M, Lefranc F, Kiss R. Galectin-1: a small protein with major functions. Glycobiology 2006; 16: 137R-157R.

13. Kovacs-Solyom F, Blasko A, Fajka-Boja R, Katona RL, Vegh L, Novak J et al. Mechanism of tumor cell-induced T-cell apoptosis mediated by galectin-1. Immunol Lett 2010; 127: 108-118.

14. Paz A, Haklai R, Elad-Sfadia G, Ballan E, Kloog Y. Galectin-1 binds oncogenic H-Ras to mediate Ras membrane anchorage and cell transformation. Oncogene 2001; 20: 7486-7493.

15. Huang EY, Chen YF, Chen YM, Lin IH, Wang CC, Su WH et al. A novel radioresistant mechanism of galectin-1 mediated by $\mathrm{H}$-Ras-dependent pathways in cervical cancer cells. Cell Death Dis 2012; 3: e251.

16. Chung LY, Tang SJ, Sun GH, Chou TY, Yeh TS, Yu SL et al. Galectin-1 promotes lung cancer progression and chemoresistance by upregulating p38 MAPK, ERK, and cyclooxygenase-2. Clin Cancer Res 2012; 18: 4037-4047.

17. Vogt PK. Jun, the oncoprotein. Oncogene 2001; 20: 2365-2377. 
18. Huang LW, Garrett AP, Bell DA, Welch WR, Berkowitz RS, Mok SC. Differential expression of matrix metalloproteinase- 9 and tissue inhibitor of metalloproteinase-1 protein and mRNA in epithelial ovarian tumors. Gynecol Oncol 2000; 77: 369-376.

19. Symowicz J, Adley BP, Gleason KJ, Johnson JJ, Ghosh S, Fishman DA et al. Engagement of collagen-binding integrins promotes matrix metalloproteinase-9-dependent E-cadherin ectodomain shedding in ovarian carcinoma cells. Cancer Res 2007; 67: 2030-2039.

20. van den Brule F, Califice S, Garnier F, Fernandez PL, Berchuck A, Castronovo V. Galectin-1 accumulation in the ovary carcinoma peritumoral stroma is induced by ovary carcinoma cells and affects both cancer cell proliferation and adhesion to laminin-1 and fibronectin. Lab Invest 2003; 83: 377-386.

21. Marshall CJ. Specificity of receptor tyrosine kinase signaling: transient versus sustained extracellular signal-regulated kinase activation. Cell 1995; 80: 179-185.

22. Cazanave SC, Elmi NA, Akazawa Y, Bronk SF, Mott JL, Gores GJ. CHOP and AP-1 cooperatively mediate PUMA expression during lipoapoptosis. Am J Physiol Gastrointest Liver Physiol 2010; 299: G236-G243.

23. Fu L, Balasubramanian M, Shan J, Dudenhausen EE, Kilberg MS. Auto-activation of c-JUN gene by amino acid deprivation of hepatocellular carcinoma cells reveals a novel C-JUN-mediated signaling pathway. J Biol Chem 2011; 286: 36724-36738.

24. Gingis-Velitski S, Loven D, Benayoun L, Munster M, Bril R, Voloshin T et al. Host response to short-term, single-agent chemotherapy induces matrix metalloproteinase-9 expression and accelerates metastasis in mice. Cancer Res 2011; 71: 6986-6996.

25. Kupferman ME, Fini ME, Muller WJ, Weber R, Cheng Y, Muschel RJ. Matrix metalloproteinase 9 promoter activity is induced coincident with invasion during tumor progression. Am J Pathol 2000; 157: 1777-1783.

26. Shibata K, Kikkawa F, Nawa A, Thant AA, Naruse K, Mizutani S et al. Both focal adhesion kinase and c-Ras are required for the enhanced matrix metalloproteinase 9 secretion by fibronectin in ovarian cancer cells. Cancer Res 1998; 58: 900-903.

27. Kim KC, Lee $\mathrm{CH}$. MAP kinase activation is required for the MMP-9 induction by TNF-stimulation. Arch Pharm Res 2005; 28: 1257-1262.

28. Abbas T, Dutta A. p21 in cancer: intricate networks and multiple activities. Nat Rev Cancer 2009; 9: 400-414.

29. Auer KL, Park JS, Seth P, Coffey RJ, Darlington G, Abo A et al. Prolonged activation of themitogen-activated protein kinase pathway promotes DNA synthesis in primary hepatocytes from p21Cip-1/WAF1-null mice, but not in hepatocytes from p16INK4a-null mice. Biochem J 1998; 336: 551-560.

30. Adams JM, Cory S. The Bcl-2 protein family: arbiters of cell survival. Science 1998; 281: 1322-1326.

31. Yang X, Zheng F, Xing H, Gao Q, Wei W, Lu Y et al. Resistance to chemotherapy-induced apoptosis via decreased caspase-3 activity and overexpression of antiapoptotic proteins in ovarian cancer. J Cancer Res Clin Oncol 2004; 130: 423-428.

32. Perego P, Righetti SC, Supino R, Delia D, Caserini C, Carenini N et al. Role of apoptosis and apoptosis-related proteins in the cisplatin-resistant phenotype of human tumor cell lines. Apoptosis 1997; 2: 540-548.

33. Wang $Y$, Wang $X$, Zhao $H$, Liang $B$, Du Q. Clusterin confers resistance to TNF-alphainduced apoptosis in breast cancer cells through NF-kappaB activation and Bcl-2 overexpression. J Chemother 2012; 24: 348-357.

34. Huang Z, Lei X, Zhong M, Zhu B, Tang S, Liao D. Bcl-2 small interfering RNA sensitizes cisplatin-resistant human lung adenocarcinoma A549/DDP cell to cisplatin and diallyl disulfide. Acta Biochim Biophys Sin (Shanghai) 2007; 39: 835-843.

35. Boucher MJ, Morisset J, Vachon PH, Reed JC, Laine J, Rivard N. MEK/ERK signaling pathway regulates the expression of $\mathrm{Bcl}-2, \mathrm{Bcl}-\mathrm{X}(\mathrm{L})$, and $\mathrm{Mcl}-1$ and promotes survival of human pancreatic cancer cells. J Cell Biochem 2000; 79: 355-369.

36. Yang $\mathrm{Y}$, Jiang $\mathrm{H}, \mathrm{Gao} \mathrm{H}$, Kong J, Zhang $\mathrm{P}, \mathrm{Hu} \mathrm{S}$ et al. The monoclonal antibody $\mathrm{CH} 12$ enhances the sorafenib-mediated growth inhibition of hepatocellular carcinoma xenografts expressing epidermal growth factor receptor variant III. Neoplasia 2012; 14: 509-518.

37. Wang $\mathrm{H}$, Zhou M, Shi B, Zhang Q, Jiang H, Sun $Y$ et al. Identification of an exon 4-deletion variant of epidermal growth factor receptor with increased metastasis-promoting capacity. Neoplasia 2011; 13: 461-471.

(1) () () Cell Death and Disease is an open-access journal published by Nature Publishing Group. This work is licensed under the Creative Commons Attribution-NonCommercial-No Derivative Works 3.0 Unported License. To view a copy of this license, visit http://creativecommons.org/licenses/by-nc-nd/3.0/

Supplementary Information accompanies this paper on Cell Death and Disease website (http://www.nature.com/cddis) 